\title{
Neuroglobin Is an Endogenous Neuroprotectant for Retinal Ganglion Cells against Glaucomatous
} Damage

\author{
Xin Wei, ${ }^{* \dagger}$ Zhanyang Yu, ${ }^{\ddagger}$ Kin-Sang Cho, ${ }^{*}$ \\ Huihui Chen, ${ }^{*}$ Muhammad Taimur A. Malik, ${ }^{*}$ \\ Xiaoming Chen, ${ }^{\dagger}$ Eng H. Lo, ${ }^{\ddagger}$ Xiaoying Wang, ${ }^{\ddagger}$ \\ and Dong F. Chen ${ }^{\star \S}$

\begin{abstract}
From the Schepens Eye Research Institute," Department of Ophthalmology, Massachusetts Eye and Ear, Harvard Medical School, Boston, Massachusetts; the Department of Ophthalmology and Ophthalmic Laboratories, ${ }^{\dagger}$ West China Hospital, Sichuan Laboratory, ${ }^{\ddagger}$ Department of Neurology and Radiology, Massachusetts General Hospital, Boston, Massachusetts; and the Center for Innovative Visual Rehabilitation, ${ }^{\S}$ VA Boston Healthcare System, Boston, Massachusetts
\end{abstract} \\ University, Chengdu, China; the Neuroprotection Research
}

Neuroglobin (NGB), a newly discovered member of the globin superfamily, may regulate neuronal survival under hypoxia or oxidative stress. Although NGB is greatly expressed in retinal neurons, the biological functions of $\mathrm{NGB}$ in retinal diseases remain largely unknown. We investigated the role of NGB in an experimental model of glaucoma, a neurodegenerative disorder that usually involves elevation of intraocular pressure (IOP). Elevated IOP is thought to induce oxidative stress in retinal ganglion cells (RGCs), thereby causing RGC death and, eventually, blindness. We found that NGB plays a critical role in increasing RGC resistance to ocular hypertension and glaucomatous damage. Elevation of IOP stimulated a transient up-regulation of endogenous NGB in RGCs. Constitutive overexpression of NGB in transgenic mice prevented RGC damage induced by glutamate cytotoxicity in vitro and/or by chronic IOP elevation in vivo. Moreover, overexpression of NGB attenuated ocular hypertension-induced superoxide production and the associated decrease in ATP levels in mice, suggesting that NGB acts as an endogenous neuroprotectant to reduce oxidative stress and improve mitochondrial function, thereby promoting RGC survival. Thus, NGB may modulate RGC susceptibility to glaucomatous neural damage. Manipulating the expression and bioactivity of NGB may represent a novel therapeutic strategy for glaucoma. (Am J Pathol 2011, 179:2788-2797; DOI: 10.1016/j.ajpath.2011.08.015)

Neuroglobin (NGB) is a novel member of the globin superfamily that is distantly related to hemoglobin and myoglobin. ${ }^{1} \mathrm{~A}$ highly conserved protein in evolution, human and mouse $\mathrm{Ngb}$, both comprised of 151 amino acids, are 94\% identical. ${ }^{2,3}$ In mammals, expression of NGB is found in the brain, retina, and other nerve tissues, predominantly in neurons but absent from glial cells. ${ }^{4-7}$ Distribution of NGB protein in the normal human retina is very similar to what has been described in mice: NGB is detected primarily in the plexiform layers and photoreceptor inner segments, which are rich in mitochondria and synapses and consume high amounts of oxygen. ${ }^{8,9}$ NGB has been shown to act as an endogenous neuroprotective molecule that enhances neuronal survival under hypoxic-ischemic insults in the brain ${ }^{10-12}$; however, its physiological functions and molecular mode of actions are not fully understood. Of note, NGB is present at a 100-fold greater concentration in the retina than in the brain, ${ }^{8,13-15}$ suggesting that the retina may be its most important site of function; however, among the many unknown roles of NGB, functional significance of NGB expression in the retina has been largely unexplored.

Supported in part by grants from the NIH National Eye Institute (R01EY017641), the NIH National Institute of Drug Abuse (R21DA024803), the Department of Veterans Affairs (5I01RX000110), the Department of Defense (W23RYX-9104-N603), and the American Health Foundation (G2007-058 to D.F.C.), and the National Institute of Neurological Disorder and Stroke (R01NS49476 to X.W.).

Accepted for publication August 18, 2011

X.W., Z.Y., and K-S.C. contributed equally to the present work.

Address reprint requests to Dong Feng Chen, M.D., Ph.D., Schepens Eye Research Institute, Department of Ophthalmology, Harvard Medical School, 20 Staniford St., Boston, MA 02114, or to Xiaoying Wang, M.D., Ph.D., Neuroprotection Research Laboratory, Department of Neurology and Radiology, Massachusetts General Hospital, Harvard Medical School, 149 13th St., Room 2411A, Charlestown, MA 02129. E-mail: dongfeng.chen@schepens.harvard.edu or wangxi@helix.mgh.harvard.edu. 
Like hemoglobin, NGB features an iron-containing central heme and an $\alpha$-helical polypeptide that is foldwrapped around the heme group and covalently attached to a histidine residue. ${ }^{16}$ NGB is reported to colocalize to the mitochondria ${ }^{8}$ and to reversibly bind oxygen, likely to supply oxygen to neurons. ${ }^{17}$ NGB has also been proposed to play a role in scavenging reactive oxygen species (ROS), ${ }^{18-20}$ modulating nitric oxide homeosta$\mathrm{sis}^{21}$ and serving as a hypoxia sensor and initiating a signal cascade ${ }^{22,23}$ in neurons. Enhanced NGB expression inversely correlates with the severity of histological and functional deficits after ischemic stroke, which supports its neuroprotective action. ${ }^{24-27}$ Although NGB expression is increased in the retina of glaucoma patients, ${ }^{9}$ the roles of NGB in the retina and in retinal neurodegenerative disorders, such as glaucoma, remain unknown. Elucidating the functional significance of NGB in the retina may uncover novel therapeutic targets for protecting RGCs and preventing vision loss caused by glaucoma or other neurodegenerative diseases.

One of the leading causes of blindness, glaucoma affects 70 million people worldwide (http://www.glaucoma. org/news/glaucoma-awareness-month.php, last accessed October 12, 2011). Increased IOP is an important risk factor. Lowering IOP slows down the progression of glaucomatous damage; however, clinical evidence suggests that glaucoma patients vary greatly in their susceptibility to elevated IOP. ${ }^{28-30}$ Several factors, including oxidative stress, ${ }^{31}$ mitochondrial dysfunction, ${ }^{32}$ and glutamate neurotoxicity ${ }^{33,34}$ are proposed to work concomitantly in the pathogenesis of the disease and in regulating RGC susceptibility to glaucomatous damage. ${ }^{35,36}$ Given possible involvement of NGB in these cellular functions, we hypothesized that NGB plays a role in glaucoma and may represent a novel target for treatment of the disease. Recently, we and others developed a convenient method of reproducibly inducing chronic IOP elevation in mice by anterior chamber injection of polystyrene microbeads. ${ }^{37,38}$ With this mouse model, we investigated the functions of NGB in the retina and, using mouse genetic tools, demonstrated a role of NGB in promoting RGC survival by reducing oxidative stress and improving mitochondrial function in glaucoma. These findings offer a unique opportunity for neuroprotective interventions aimed at effective control of neuronal damage in glaucoma.

\section{Materials and Methods}

\section{Ngb Transgenic Mice}

$\mathrm{Ngb}$ transgenic mice (Ngb-Tg), driven under a cytomegalovirus (CMV) promoter, were generated according to a standard procedure. In brief, full-length cDNA sequence of mouse Ngb (GenBank accession no. NM_022414) was cloned and fused with $\mathrm{N}$-terminal hemagglutinin epitope tag. The pCMV-Ngb DNA fragment was used to generate Ngb-Tg mice in the Transgenic Core Facility at Massachusetts General Hospital. ${ }^{39}$ The mice were initially created in a B6C3F1 background and backcrossed to
C57BL/6J mice for more than nine generations. ${ }^{39} \mathrm{PCR}$ analysis using primers flanking $\mathrm{CMV}$ and $\mathrm{Ng} b$ sequences detected a 940-bp DNA product and confirmed the presence of mouse Ngb transgene. Western blot and quantitative RT-PCR analyses showed a greater than twofold increase of $\mathrm{Ngb}$ protein and mRNA levels in the brain tissues of Ngb-Tg mice, compared with wild-type littermates. Increased expression of $\mathrm{Ngb}$ was found in both neurons and astrocytes in Ngb-Tg mouse brains, consistent with the expression pattern driven by a non-tissuespecific CMV promoter. ${ }^{39}$ In the present study, adult mice ( $>2$ months of age) overexpressing $\mathrm{Ngb}$ and their wild-type littermates were used. The experimental procedures and use of animals were approved and monitored by the Animal Care Committee of the Schepens Eye Research Institute and of Massachusetts General Hospital and conformed to standards of the Association for Research in Vision and Ophthalmology.

\section{Induction of Chronic IOP Elevation in Mice}

The procedure for inducing chronic IOP elevation and glaucoma in mice was essentially as described previously. ${ }^{38}$ Briefly, mice were anesthetized by intraperitoneal injection of a ketamine $(120 \mathrm{mg} / \mathrm{kg})$ and xylazine $(12$ $\mathrm{mg} / \mathrm{kg}$ ) mixture (Phoenix Pharmaceutical, St. Joseph, $\mathrm{MO})$ supplemented by topical proparacaine $\mathrm{HCl}(0.5 \%$; Bausch \& Lomb Pharmaceuticals, Tampa, FL). Elevation of IOP was induced unilaterally in adult wild-type C57BL/6J and Ngb-Tg mice by an anterior chamber injection of $10-\mu \mathrm{m}$ polystyrene microbeads (Molecular Probes; Invitrogen, Eugene, OR) that had been resuspended in PBS at a final concentration of $7.2 \times 10^{6}$ beads $/ \mathrm{mL}{ }^{38}$ The right cornea was gently punctured with a 30-gauge needle without injuring the lens or iris. Through this entry wound, $2 \mu \mathrm{L}$ of microbeads were injected into the anterior chamber using a glass micropipette. At week 4, a second injection of microbeads was made to maintain the prolonged IOP elevation. Mice of the control group received an injection of $2 \mu \mathrm{L}$ of PBS into the anterior chamber. Mice exhibiting corneal opacity or signs of inflammation (eg, cloudy anterior chamber, cellular debris in the lens, fibrin strands, or pigment dispersion) were excluded from further study.

\section{IOP Measurement}

After the injection of microbeads, IOP was measured every other day in both eyes, using a TonoLab tonometer (Colonial Medical Supply, Franconia, NH) as described previously. ${ }^{38}$ The measurement of IOP was conducted consistently at the same time in the morning. Mice were anesthetized by isoflurane inhalation ( $2 \%$ to $4 \%$; Webster Veterinary, Devens, MA) that was delivered in $100 \%$ oxygen in a precision vaporizer. Measurement was initiated within 2 to 3 minutes after the animal lost consciousness. Six measurements were obtained from each eye, and the mean value was calculated to determine the IOP. 


\section{Quantification of Ngb Expression with RT-qPCR and Western Blot}

Procedures for RT-qPCR and Western blot were performed as described previously. ${ }^{40}$ To investigate $\mathrm{Ngb}$ mRNA and protein expression, mice $(n>4$ in each experimental group per time point) were sacrificed on day 0,3 , and 7 after microbead injection. Individual retinas were isolated and homogenized using a rotor-stator homogenizer. RNAs were extracted with an RNeasy mini kit (Qiagen, Valencia, CA) according to the manufacturer's instructions. Reverse transcription was performed using Moloney murine leukemia virus reverse transcriptase (MMLV-RT) (Invitrogen, Carlsbad, CA) to obtain cDNA. In a nuclease-free microcentrifuge tube, $1 \mu \mathrm{L}$ of $50 \mu \mathrm{mol} / \mathrm{L}$ oligo(dT) $)_{20}, 11 \mu \mathrm{L}$ of total RNA ( $1 \mathrm{ng}$ to $5 \mu \mathrm{g}$ total RNA), and $1 \mu \mathrm{L}$ of $10 \mathrm{mmol} / \mathrm{L}$ dNTP mix were added into $12 \mu \mathrm{L}$ sterile distilled water. The mixture was heated at $65^{\circ} \mathrm{C}$ for 5 minutes and quickly chilled on ice. After brief centrifugation, $4 \mu \mathrm{L}$ of $5 \times$ first-strand buffer and $2 \mu \mathrm{L}$ of $0.1 \mathrm{~mol} / \mathrm{L}$ dithiothreitol were added to the tube and then incubated at $42^{\circ} \mathrm{C}$ for 2 minutes, followed by addition of $1 \mu \mathrm{L}(200$ units) of M-MLV-RT. The tube was then incubated at $42^{\circ} \mathrm{C}$ for 50 minutes, and the reaction was inactivated by heating at $70^{\circ} \mathrm{C}$ for 15 minutes. Real-time PCR was performed on an ABI Prism 7000 sequence detection systems (Applied Biosystems, Foster City, CA). The primer sequences for detecting $\mathrm{Ngb}$ were forward primer: 5'-TACAATGGCCGCCAGTTCT-3' and reverse primer: 5'TGGTCACTGCAGCATCAATCA-3'.

Data were analyzed according to the comparative threshold cycle $\left(\mathrm{C}_{\mathrm{t}}\right)$ method and were normalized to GAPDH expression. Melting curves of each PCR reaction were generated to ensure the purity of the amplification product.

For Western blot analysis, individual retinas were lysed in lysis buffer; protein concentration was measured with a Micro BCA protein assay reagent kit (Pierce; Thermo Fisher Scientific, Rockford, IL), and $100 \mu \mathrm{g}$ of protein from each sample was loaded onto a SDS gel for electrophoresis and transferred to nitrocellulose membranes. The blots were reacted with primary antibodies against NGB (chicken anti-human neuroglobin, 1:2000; BioVendor, Candler, NC) and actin (mouse anti-actin, 1:5000, Chemicon; Millipore, Billerica, MA) as a control in a solution containing $0.05 \%$ Tween-20, 1\% bovine serum albumin, and $4 \%$ nonfat dry milk at $4^{\circ} \mathrm{C}$ overnight. The membrane was incubated with the corresponding horseradish peroxidaseconjugated secondary antibody $(1: 10,000)$ for 1 hour at room temperature. An enhanced chemiluminescence system (Pierce; Thermo Fisher Scientific) was used for antibody detection. All experiments were performed in triplicate.

\section{Detection of Ngb Expression with Immunohistochemistry}

Mice were sacrificed at day $0,3,7,28$, and 56 after PBS or microbead injection and were transcardially perfused with saline. Eyeballs were removed, postfixed in $4 \%$ paraformaldehyde overnight, and flat-mounted or cryoprotected with 30\% sucrose, followed by cryosection to 14- $\mu \mathrm{m}$ thickness. Retinal flat mounts and sections were incubated with a primary antibody against a RGC-specific marker, $\beta$-III-tubulin (Tuj-1; Sigma-Aldrich, St. Louis, $\mathrm{MO}$ ) and/or NGB protein (GenScript, Piscataway, NJ), followed by reaction with a biotin-conjugated secondary antibody. Customized polyclonal NGB antibodies were produced by immunizing rabbits with two synthetic peptides (KLH coupled, FQYNGRQFSSPEDC and IRQSWRVVSRSPLEC) corresponding to mouse Ngb and were purified by protein $A$ and peptide affinity chromatography. Immunolabeling was revealed by reaction with streptavidin, Alexa Fluor 488 (Invitrogen), or Alexa Fluor 546 (Invitrogen) conjugate. Six to eight random, nonoverlapping fields (1176 $\mu \mathrm{m}^{2}$ each) containing the optic nerve head were selected from each retinal section. Tuj-1-positive cells were counted as described previously. ${ }^{38,40}$ At least three nonoverlapping retinal sections of each eye were studied. Values are reported as means $\pm S D$.

\section{Examination of NGB Function in Purified RGC Cultures}

To isolate RGCs from the mouse retina, we used a magnetic-bead separation method with a primary antibody against a RGC-specific cell surface marker, Thy $1.2 .{ }^{41}$ In brief, retinas taken from postnatal day 2 (P2) mouse pups ( $n=4$ per group) were dissected and dissociated by incubation with $1 \%$ papain (Worthington Biochemical, Lakewood, NJ) in Hank's buffered saline solution containing $5 \mathrm{U} / \mathrm{mL}$ DNase (Gibco; Invitrogen) for 10 minutes at $37^{\circ} \mathrm{C}$. Dissociated cells were transferred to a solution containing the papain inhibitor ovomucoid (1\%; Worthington Biochemical) and were triturated. Cells were then reacted with a rat anti-Thy 1.2 antibody conjugated to micrometal beads (CD90; Miltenyi Biotec, Auburn, CA) in an elution buffer (PBS with $0.5 \%$ bovine serum albumin and $2 \mathrm{mmol} / \mathrm{L}$ EDTA; Sigma-Aldrich). Cell suspensions were loaded onto a metal column and separated with the elution buffer in the presence and absence of a magnetic field. Isolated RGCs were seeded in 24-well plates coated with poly-D-lysine ( $10 \mu \mathrm{g} / \mathrm{mL}$; Sigma-Aldrich) and laminin (10 $\mu \mathrm{g} / \mathrm{mL}$; Sigma-Aldrich) and were cultured in neurobasal medium supplemented with B27 Serum Free Supplement as described previously. ${ }^{42}$ To confirm cell identity, cultures were stained with the RGCspecific antibody Tuj-1. Cell viability in the presence of different concentrations of glutamate $(0,5,7.5$, and 10 $\mathrm{mmol} / \mathrm{L}$ ) was determined with a live/dead assay (Invitrogen). Ten to twelve randomly selected nonoverlapping fields were photographed from each well, and both live and dead cells were counted by observers without knowledge of the experimental group. Cells bearing neurites were counted under a Nikon TE300 inverted fluorescence microscope equipped with fluorescent illumination. 

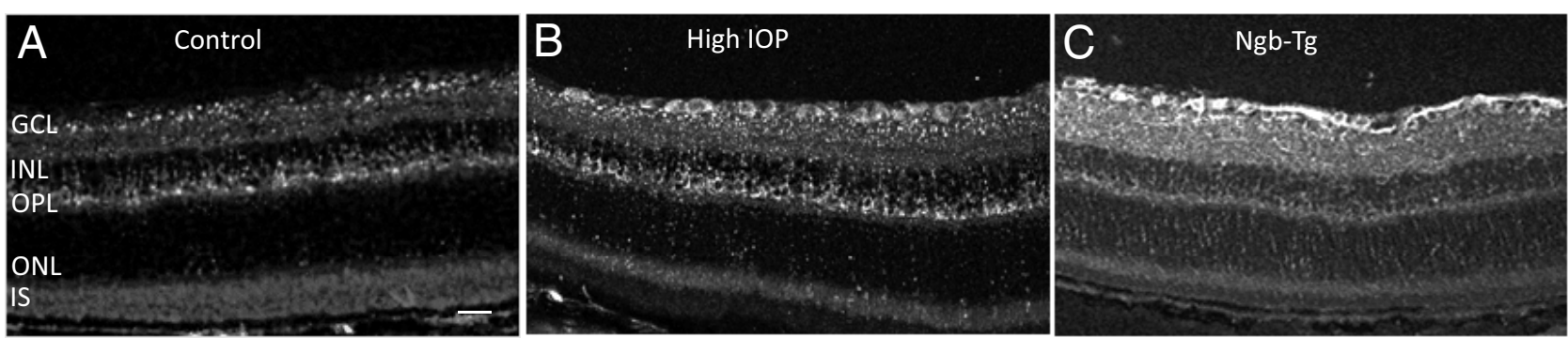

Figure 1. Ngb up-regulation in response to elevated intraocular pressure (IOP) in the mouse retina. A-C: Representative epifluorescence photomicro-

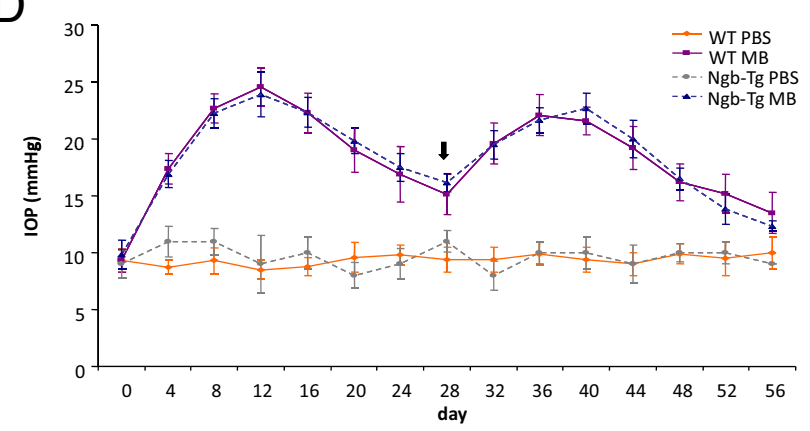
graphs of Ngb immunolabeling in retinal sections of an untreated wild-type mouse (A), mouse at 3 days after IOP elevation (B), and that of untreated Ngb-Tg mouse (C). Elevated IOP-induced up-regulation of Ngb is noted primarily in the GCL of wild-type mice. GCL, ganglion cell layer; INL, inner nuclear layer; IS, inner segment of photoreceptor; ONL, outer nuclear layer; OPL, outer plexiform layer. Scale bar $=20 \mu \mathrm{m}$. D: IOP elevation after injection of microbeads into anterior chamber. In WT mice (solid lines), the IOP of PBS-injected eyes of WT mice $(n=12)$ remained steady at $10.7 \pm 1.8$ $\mathrm{mmHg}$. Injection of $10-\mu \mathrm{m}$ microbeads (MB; $n=6)$ induced IOP elevation for approximately 28 days; a second injection of MB (arrow) extended the IOP elevation up to 8 weeks. In Ngb-Tg mice (dashed lines), injection of PBS $(n=6)$ or microbeads $(n=6)$ into the anterior chamber induced a kinetics of IOP elevation similar to that of wild-type mice. Data are presented as means $\pm \mathrm{SD}$

\section{Measurement of Superoxides Using Dihydroethidium Staining}

Dihydroethidium (DHE) staining (Sigma-Aldrich) was used to evaluate the in situ levels of superoxides in the retina. Retinal sections were incubated with $25 \mathrm{nmol} / \mathrm{L}$ $\mathrm{DHE}$ at $37^{\circ} \mathrm{C}$ for 25 minutes in a humidified and darkened chamber. Fluorescent images of ethidium bromide were obtained using a T300 microscope (Nikon, Hong Kong) equipped with an epi-fluorescence microscope. Fluorescence densities of DHE stain were quantified using ImageJ software version 1.44 (NIH, Bethesda, MD).

\section{Measurement of ATP Levels}

A primary function of mitochondria is to generate ATP through oxidative phosphorylation. The ATP level of individual mouse retina was assessed in fresh retinas using a commercial kit (Promega Turner BioSystems, Sunnyvale, $\mathrm{CA})$. The mouse retina dissected in ice-cold saline was transferred quickly to $0.25 \mathrm{~mL}$ cold $5 \%$ perchloric acid. After tissue homogenization, the tissue was centrifuged at $10,000 \times g$ for 10 minutes. A $10-\mu \mathrm{L}$ aliquot of the clear supernatant was diluted 200 -fold with distilled water, and a $50-\mu \mathrm{L}$ sample of the diluents was taken for measuring the ATP content using the luciferin-luciferase method under a luminometer (Promega Turner BioSystems). Values of tissue ATP contents were determined according to the standard curve generated using a series of known ATP concentrations, normalized to the protein levels.

\section{Statistical Analysis}

Statistical significance of data obtained from immunoblot, RT-qPCR, and cell counting was determined by analysis of variance using StatView software version $4.11 \mathrm{~J}$ for
Macintosh (SAS Institute, Cary, NC). Significance was set at $P<0.05$. All values are expressed as means $\pm \mathrm{SD}$.

\section{Results}

\section{NGB Expression Profile in Response to IOP Elevation}

Defining the expression profiles of NGB under normal and diseased conditions of the retina is a first step toward understanding the roles of NGB in retinal neurons. Consistent with other reports, ${ }^{8,13-15}$ we detected moderate expression of $\mathrm{NGB}$ in the ganglion cell layer (GCL), inner plexiform layer (IPL), outer plexiform layer (OPL), and photoreceptor inner segment in retinal sections of adult mice using immunofluorescence labeling (Figure 1, A-C). Taking advantage of our newly developed mouse model of glaucoma, we also examined the expression of NGB in the mouse retina under a diseased condition. IOP elevation was induced unilaterally by anterior chamber injection of polystyrene microbeads, as described previously ${ }^{38}$; mice receiving unilateral injection of PBS in the anterior chamber served as controls (Figure 1D). Injection of PBS into the anterior chamber did not affect the IOP level (10.7 \pm 1.8 $\mathrm{mmHg}$ ), compared with the noninjected group (9.8 \pm $2.1 \mathrm{mmHg}) .{ }^{38}$ A single injection of microbeads induced elevation of IOP for 4 weeks at a peak level of $24.2 \pm$ $2.3 \mathrm{mmHg}$, and a second injection maintained the elevation of IOP to 8 weeks (Figure 1D). Overexpression of NGB did not affect the basal level of IOP, and injection of microbeads in $\mathrm{Ngb}-\mathrm{Tg}$ mice induced a kinetic of IOP elevation similar to that of the wild-type littermates (Figure 1D). However, elevated IOP resulted in an increase in NGB expression in the retina, including the $\mathrm{GCL}, \mathrm{INL}$, and $\mathrm{OPL}$, in wild-type mice (Figure 1B), 

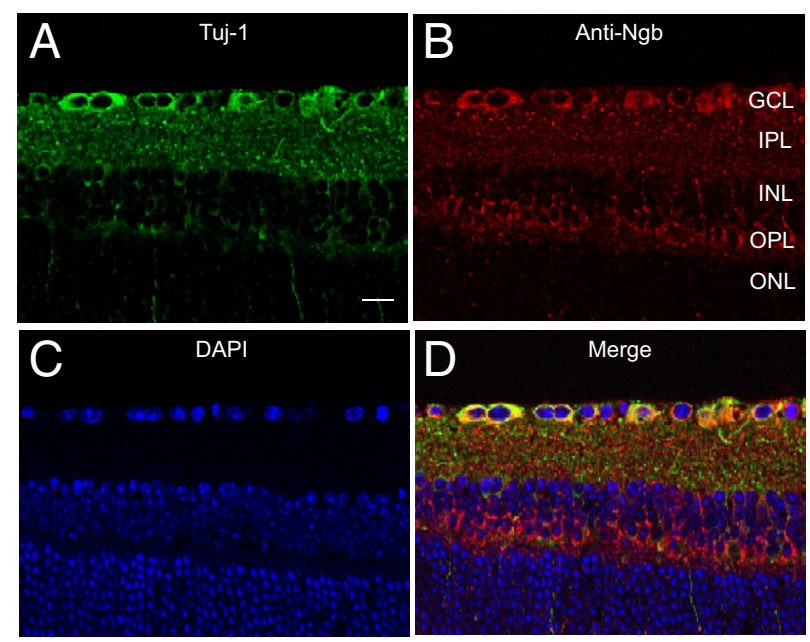

Figure 2. Transient increase of Ngb expression in RGCs induced by elevation in IOP. Representative epifluorescence photomicrographs of a retinal section taken from WT mice at 3 days after induction of IOP elevation. The retinal section was triple-labeled by Tuj-1 antibody (green; RGC marker) (A), anti-Ngb (red) (B), and DAPI (blue; nuclear marker) (C). Note the intensive Ngb immunolabeling in the GCL, colocalizing with the immunolabeling of the RGC marker Tuj-1 in the merged image (D). Scale bar $=10 \mu \mathrm{m}$.

relative to expression in PBS-injected eyes. Immunolabeling of NGB in Ngb-Tg mice also confirmed high levels of NGB expression in the GCL and other retinal layers (Figure 1C).

Because RGCs are the primary cell type affected in glaucoma, we asked whether elevated IOP increased NGB expression in RGCs specifically. Using double immunolabeling with primary antibodies against NGB and the RGC specific marker anti- $\beta$-III-tubulin (Tuj-1), we examined the expression profile of NGB after IOP elevation. Mice were sacrificed at $0,3,7,14$, and 56 days after anterior chamber injection of microbeads, and retinal sections were prepared and subjected to double immunolabeling. Enhanced expression of NGB colocalized with Tuj-1 labeling (Figure 2). Apparent increase of NGB immunofluorescence expression was consistently noted in RGCs on day 3 after IOP elevation (Figure 2), but became unapparent thereafter (data not shown). This temporal pattern of NGB up-regulation appeared to inversely correlate with elevated-IOP-induced RGC death, which became significantly detectable after day 7 of IOP elevation, supporting a role for endogenous NGB in promoting $R G C$ resistance to glaucomatous damage.

We next corroborated this finding using quantitative realtime RT-PCR and Western blot analysis. Mice were sacrificed at 0, 3, and 7 days after anterior chamber injection of microbeads and induction of IOP elevation, and retinal RNAs or proteins were extracted. Elevation of IOP induced a twofold increase in NGB protein levels in the mouse retinas at 3 and 7 days after IOP elevation (Figure $3, A$ and $B$ ). In agreement with these data, significant increase of NGB mRNA levels was also observed in the mouse retina at both 3 and 7 days after IOP elevation, compared with the control group (Figure 3C). Taken together, these data indicate that NGB is expressed in RGCs of the normal retina, and that its expression is significantly up-regulated in RGCs and other retinal layers after the elevation of IOP. The data thus sug- gest a potential role for NGB in glaucomatous RGC damage.

\section{Neuroprotective Effect of NGB under Elevated IOP}

To uncover the functional roles of NGB in the retina and to test whether expression of NGB increases RGC resistance to glaucomatous damage, we applied genetic tools to investigate RGC survival under normal and diseased conditions in Ngb-Tg mice. Increased expression of NGB protein in the retina and RGCs of $\mathrm{Ngb}-\mathrm{Tg}$ mice was demonstrated both by Western blot (Figure 3, A and B) and real-time RT-PCR (Figure $3 \mathrm{C}$ ). There was an approximately twofold increase of NGB proteins in retinas of $\mathrm{Ngb}$-Tg mice, compared with WT mouse retinas (Figure $3 \mathrm{~B})$, similar to what has been reported in brain. ${ }^{39}$ According to these data (Figure 3, $A-C$ ), elevation of IOP did not induce further increase of NGB expression in retinas of $\mathrm{Ngb}-\mathrm{Tg}$ mice, compared with that of Ngb-Tg mice with a normal IOP, suggesting a ceiling effect.

RGC survival in WT and Ngb-Tg mice that received PBS or microbead injections was quantified as described previously. ${ }^{38}$ Mice were sacrificed at 3 days to 8 weeks after injection. Immunolabeling of retinal flat mounts with Tuj-1 antibody revealed RGC loss in both retinas of WT and Ngb-Tg mice after IOP elevation (Figure 4). The number and density of Tuj-1-labeled RGCs were quantitatively assessed $^{38,43}$ Counts of Tuj-1-positive RGCs indicated no significant difference between untreated control WT and

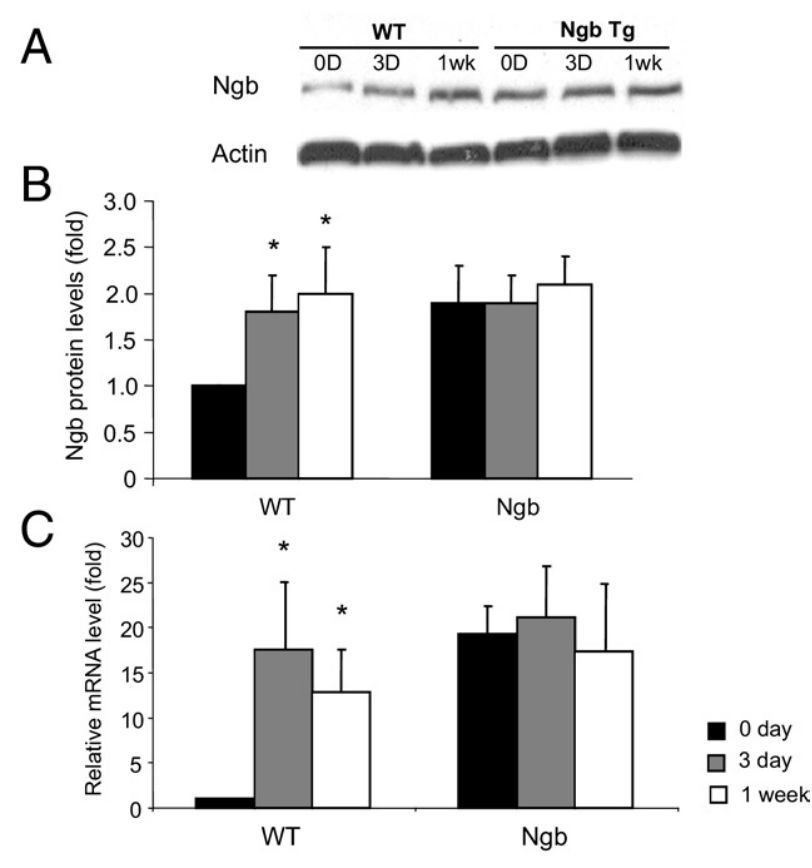

Figure 3. Comparison of Ngb expression profile in the retinas of WT and Ngb-Tg mice. A: Representative immunoblot of triplicate experiments measuring Ngb protein levels in retinas of WT and Ngb-Tg mice harvested at 0 (OD), 3 (3D) and 7 (1wk) days after anterior chamber injection of microbeads. $\mathbf{B}$ and C: Quantification of Ngb protein $(\mathbf{B})$ by Western blot and mRNA levels (C) by real-time PCR in retinas of WT and Ngb-Tg mice, before and after IOP elevation. The experiments were performed in triplicate, and values are presented as means $\pm \mathrm{SD}$. ${ }^{*} P<0.05$ (analysis of variance). $n>4$ per group. 

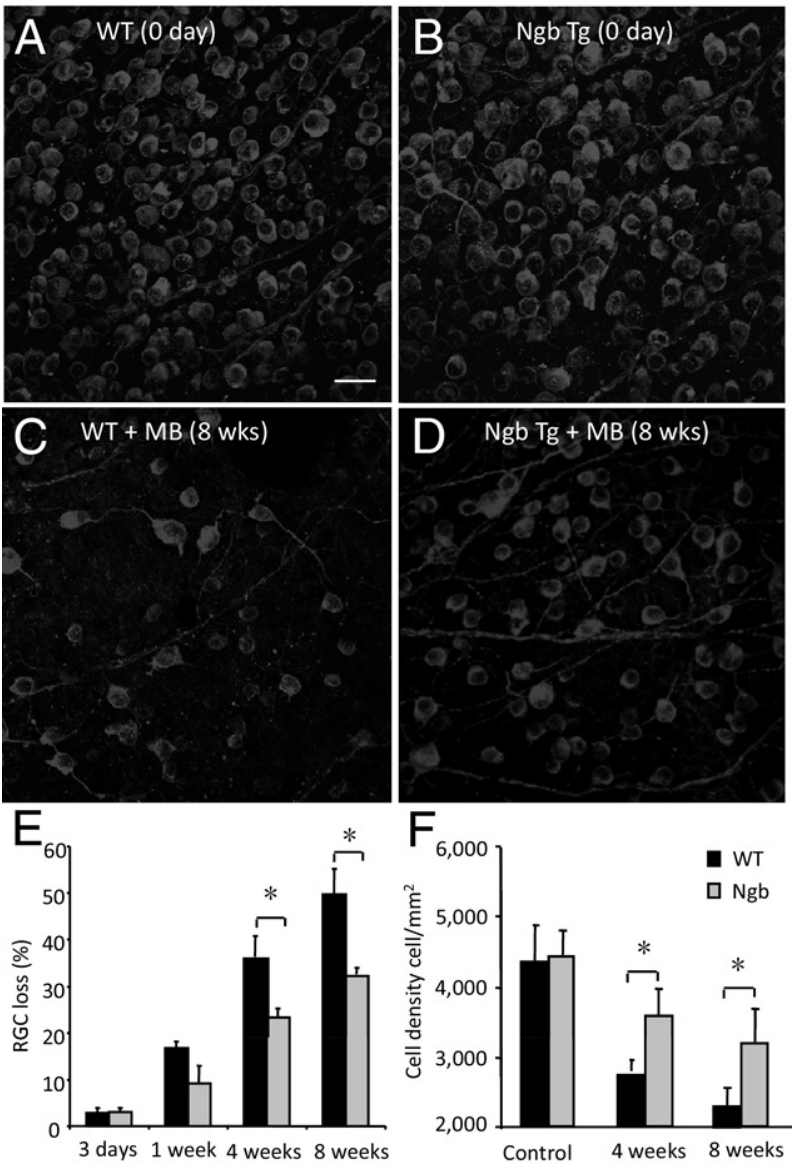

Figure 4. Neuroprotective effect of $\mathrm{Ngb}$ in an in vivo model of ocular hypertension. A-D: Representative epifluorescence photomicrographs of retinal flat mounts immunolabeled by Tuj- 1 antibody (RGC marker). The retinas were taken from the eyes of WT (A and $\mathbf{C}$ ) and Ngb-Tg (B and $\mathbf{D})$ mice that received anterior chamber injection of either control PBS (A and $\mathbf{B}$ ) or microbeads (MB) $(\mathbf{C}$ and $\mathbf{D})$. Mice were sacrificed at 8 weeks after injection. Note reduced RGC density in the retinal flat mount of WT mouse with an elevated IOP (C) and lesser reduction in RGC number in the retina of Ngb-Tg mouse $(\mathbf{D})$. Scale bar $=10 \mu \mathrm{m}$. E and $\mathbf{F}$ : Percentage of RGC loss (E) and quantification of RGC densities (F) in WT and Ngb-Tg mice at various time points after induction of IOP elevation. Significant protective effects on RGC survival after IOP elevation were noted in Ngb-Tg mice, compared with WT mice. Values are presented as means \pm SD. ${ }^{*} P<0.05$; ${ }^{* *} P<0.01$ (analysis of variance). All photos were taken under the same magnification using $40 \mathrm{x}$ objective. $n=6$ per group.

Ngb-Tg mice (data not shown), suggesting that overexpression of NGB did not affect RGC survival under the normal condition. In agreement with previous reports in other experimental models of glaucoma, ${ }^{44}$ loss of RGCs in WT mice was detectable as early as 7 days after the elevation of IOP (Figure 4E). There was a significant increase in RGC survival in Ngb-Tg mice at both 4 and 8 weeks after IOP elevation, compared with WT mice (Figure 4F). Thus, overexpression of NGB promotes RGC survival under elevated IOP, suggesting a neuroprotective role of NGB for RGCs.

\section{Neuroprotective Effect of NGB in Purified RGC Cultures}

To further probe for the mechanism and to determine whether NGB acts cell autonomously in RGCs to prevent cell death, we investigated the effect of NGB in purified
RGC cultures by taking advantage of our ability to isolate the cell population. Glutamate-induced neuroexcitotoxicity is proposed to be an important mechanism contributing to RGC loss in glaucoma. ${ }^{32,45}$ We therefore hypothesized that, if NGB acts cell autonomously as a neuroprotective protein under ocular hypertension in vivo, we would observe a neuroprotective effect of NGB against glutamate-induced neurotoxicity in purified RGC cultures. RGCs were isolated from P2 WT and Ngb-Tg mouse pups as described previously ${ }^{41}$ and were cultured under various concentrations of glutamate. As expected, increasing levels of glutamate stimulation resulted in incremental loss of RGCs in culture. Overexpression of NGB significantly enhanced survival in glutamate-treated cultures at all concentrations of glutamate treatment tested (Figure 5). Similar to observations in vivo, RGCs of $\mathrm{Ngb}-\mathrm{Tg}$ mice did not exhibit enhanced ability to survive in culture under the normal condition, when in the absence of glutamate (Figure 5C), suggesting a neuroprotective role of NGB only under the stressed conditions. These data demonstrate a cell-autonomous effect of NGB on promoting RGC survival.

\section{Amelioration of Elevated-IOP-Induced Oxidative Stress and Mitochondrial Dysfunction by NGB Overexpression}

Previous studies suggested that IOP elevation and/or glutamate stimulation induce oxidative stress and mitochondrial impairment in RGCs. ${ }^{46}$ We therefore assessed these changes in mice. Because significant loss of RGCs was detectable from day 7 after IOP elevation, oxidative
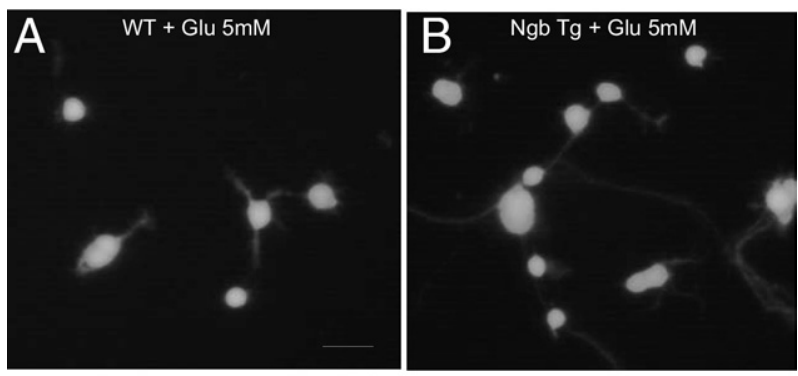

C

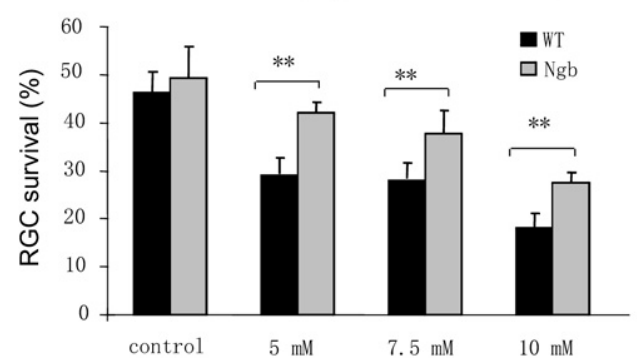

Figure 5. Neuroprotective effects of Ngb against glutamate-induced neurocytotoxicity in vitro. A and B: Representative epifluorescence photomicrographs of purified RGCs taken from P2 WT (A) and Ngb-Tg (B) mouse pups; cells were cultured for 3 days under treatment with $5 \mathrm{mmol} / \mathrm{L}$ glutamate. Scale bar $=20 \mu \mathrm{m}$. C: Quantification of RGC survival in culture. RGCs were incubated under different concentrations of glutamate $(0,5,7.5$, and $10 \mathrm{mmol} / \mathrm{L})$. Note increased survival of RGCs isolated from Ngb-Tg mice, compared with WT mice, at every concentration of glutamate treatment ( $n=6$ per group). Values are presented as means $\pm \mathrm{SD} .{ }^{* * *} P<0.01$ (analysis of variance). 
DHE
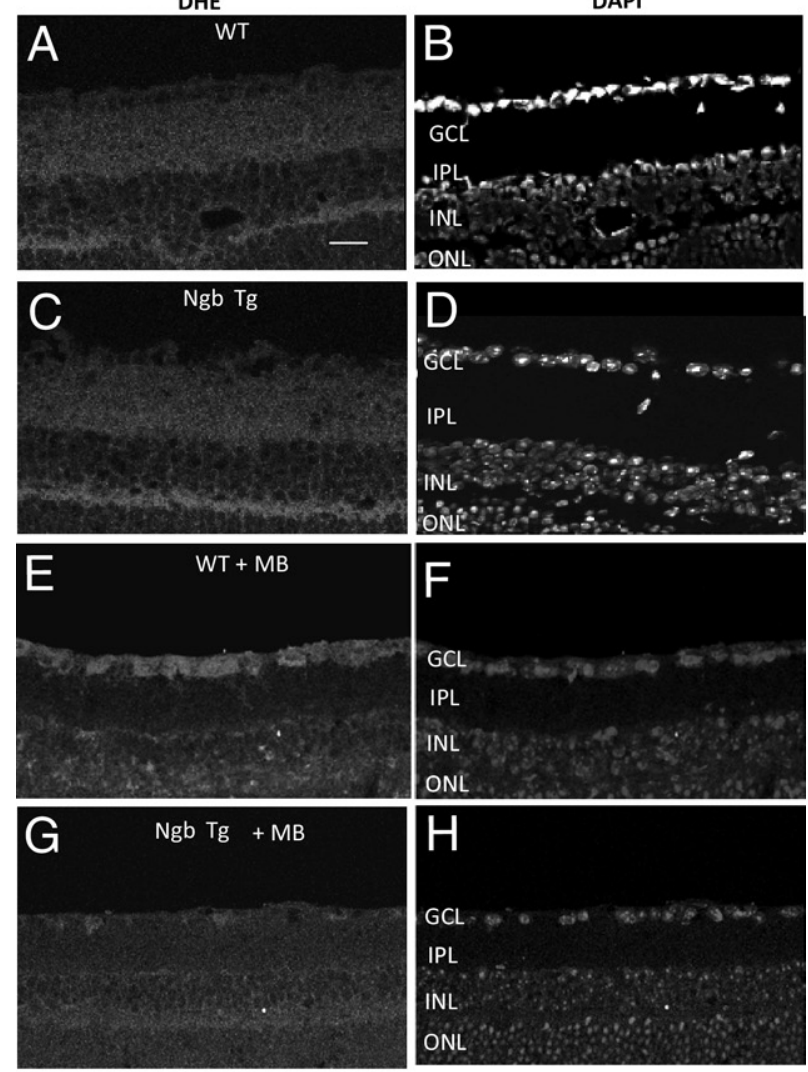

I

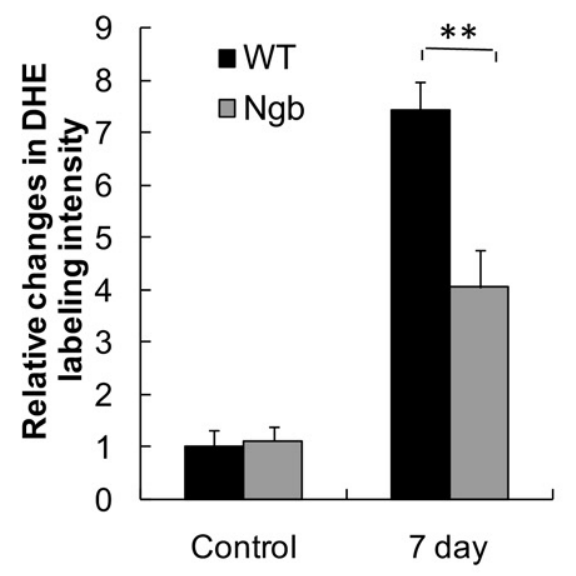

$J$

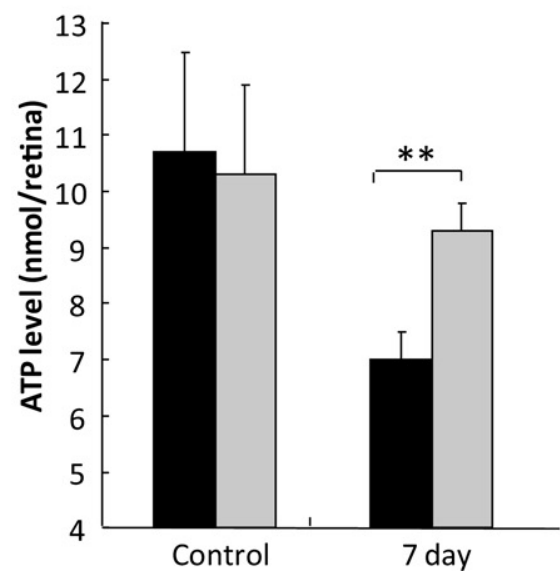

stress in the mouse retina was measured at 7 days after microbead injection by detecting the expression of superoxides as an oxidative marker, using DHE stain. ${ }^{4748}$ In the presence of superoxides, DHE is oxidized and turned into fluorescent ethidium bromide, which is trapped intracellularly by DNA intercalation. DHE is thus commonly used to evaluate in situ levels of superoxide under the oxidative conditions. In the control group, we detected a very low basal level of DHE signal in the GCL of both WT and $\mathrm{Ngb}$-Tg mice (Figure 6, A-D); no significant difference was observed between the WT and Ngb-Tg mice (Figure 6I). Induction of IOP elevation markedly increased DHE stain in the GCL of WT mice (Figure 6, $\mathrm{E}-\mathrm{H})$. There was a greater than sevenfold increase in the DHE signal in the GCL of elevated-IOP mice, relative to the normal-IOP controls, indicative of oxidative stress in RGCs after IOP elevation. In contrast, in Ngb-Tg mice, DHE levels were significantly reduced in the retinas of mice with elevated IOP, compared with those of WT mice under the similar conditions (Figure 6I), suggesting that overexpression of $\mathrm{NGB}$ reduces ROS production in RGCs under the stress condition.

Increased production of ROS and mitochondrial dysfunction or decrease in cellular ATP are closely related. We therefore assessed changes in mitochondrial function and cellular energy preservation in the retina under elevated IOP by measuring intracellular ATP levels. The retinas of WT and $\mathrm{Ngb}-\mathrm{Tg}$ mice with normal IOP revealed a similar basal level of ATPs (Figure 6J). Increased IOP in WT mice significantly diminished the cellular energy levels in the retina. There was an approximately $30 \%$ reduction of retinal ATP levels at 7 days after IOP elevation, implicating a disruption of mitochondrial function (Figure $6 \mathrm{~J})$. In contrast, overexpression of NGB in transgenic mice attenuated elevated-IOP-induced reduction of ATP levels in the retina. The ATP levels of $\mathrm{Ngb}-\mathrm{Tg}$ mice measured at 7 days after IOP elevation were similar to those of WT and Ngb-Tg mice with a normal IOP and were significantly higher than for elevated-IOP WT mice (Figure 6J). Thus, overexpression of NGB preserved mitochondrial function after IOP elevation. These results suggest that NGB protects RGCs against elevated-IOP-induced cell damage, at least in part, through reducing reactive oxidative products and preserving the cellular energy levels.

Figure 6. Overexpression of Ngb in Ngb-Tg mice attenuates elevated-IOPinduced superoxide production and its reduction of ATP levels in RGCs in vivo. A-H: Representative epifluorescence photomicrographs of retinal sections stained with DHE (red), taken from untreated eyes of WT (A and B) and Ngb-Tg (C and $\mathbf{D})$ mice or eyes of WT (E and $\mathbf{F})$ and Ngb-Tg $(\mathbf{G}$ and $\mathbf{H})$ mice with induced elevation of IOP. Retinal sections were counterstained with the nuclear marker DAPI (blue) to reveal laminar structure. DHE signal is absent in the GCL of normal WT and Ngb-Tg retinas, but is strongly up-regulated in that of elevated-IOP WT mice. I: Quantification of DHE signal in retina sections of WT (black) and Ngb-Tg (gray) mice taken from the control group, which has a normal IOP, or at 7 days after induction of IOP elevation. Scale bar $=20 \mu \mathrm{m}$. Values are presented as means $\pm \mathrm{SD}$. ${ }^{* * *} P<0.01$ (analysis of variance). $n=6$ per group. J: Overexpression of Ngb preserves ATP levels after IOP elevation. ATP levels were assessed in the retinas of WT (black) and Ngb-Tg (gray) mice under normal IOP (control) or at 7 days after anterior chamber injection of microbeads. Values are presented as means $\pm \mathrm{SD}$. ${ }^{* *} P<0.01$ (analysis of variance). $n=4$ per group. 


\section{Discussion}

With the present study, we report for the first time application of mouse transgenic technology to investigate functional significance of NGB overexpression in RGCs in glaucoma. We established several findings. First, we found that, under the normal condition, NGB is enriched in RGCs and is expressed at a much higher level in RGCs of $\mathrm{Ngb}-\mathrm{Tg}$ mice. Second, ocular hypertension induces a transient up-regulation of NGB expression in RGCs in WT mice that is inversely correlated with elevated-IOP-induced RGC death, supporting a potential neuroprotective function of NGB. Third, constitutive overexpression of NGB in Ngb-Tg mice promoted RGC survival in vivo in a hypertensive experimental model of glaucoma, demonstrating for the first time that endogenous up-regulation of NGB may be a protective mechanism in neurons confronting a stressed or diseased condition. Moreover, overexpression of NGB protected RGCs against glutamate-induced neurotoxicity in culture, an in vitro model of normotensive glaucoma, further suggesting its role as an endogenous neuroprotective agent. Finally, NGB attenuated elevated-IOP-induced superoxide production and mitochondrial dysfunction to support RGC survival. These data, from both the in vitro normotensive and in vivo hypertensive models of glaucoma, reveal a novel activity of NGB in the mouse retina. This activity strongly suggests that NGB functions as an endogenous neuroprotective protein influencing RGC susceptibility to glaucomatous neuronal damage. Concordance of the data in the in vitro and in vivo models indicates that NGB may be a valuable drug target for pharmacological interventions to treat both hypertensive and normotensive glaucoma.

Notably, the mouse retina responds to elevated IOP by up-regulating NGB expression, and this constitutive overexpression of NGB promotes RGC survival under ocular hypertension. Despite increasing knowledge of the neuroprotective function of NGB in central nervous system neurons, evidence demonstrating the change of NGB levels in response to disease conditions in the rodent brain has been lacking. Studies of cell-based assays suggested that $\mathrm{NGB}$ function is associated with $\mathrm{O}_{2}$ consumption. For example, NGB binds $\mathrm{O}_{2}$ with high affinity, and early reports pointed to a likely role in $\mathrm{O}_{2}$ transport and its involvement in neuronal adaptation to hypoxic conditions. ${ }^{12,49}$ NGB may act as a sensor of oxidative stress $^{22,23}$ or as a scavenger of nitric oxide and regulator of ROS in the brain under cerebral ischemia. ${ }^{12,24,49}$ Nonetheless, evidence showing changes of NGB levels under hypoxic or ischemic conditions in the rodent brain is sparse. Our detection of expression of NGB in the GCL, $I P L$, and OPL of a normal mouse retina is consistent with reports of others. ${ }^{13-15,50}$ In the present study, we used multiple methods to demonstrate that NGB levels are up-regulated in RGCs in vivo under a diseased condition involving oxidative stress and mitochondria dysfunction, suggesting its involvement in stress or in ocular hypertension.

Endogenous NGB was up-regulated only transiently in RGCs on day 3 after IOP elevation, and appeared to decline to a normal level thereafter (Figure 2). Consistent with this finding, RGC death was significantly detectable in the retinas of WT mice after 7 days of IOP elevation, but not earlier (Figure 5), strongly implicating a neuroprotective role for NGB in the retina. We note that the expression of NGB in Ngb-Tg mice was not stimulated by IOP elevation, suggesting that a high level of NGB expression may mediate lateral inhibition or other unknown feedback mechanisms to regulate NGB transcription and protein production. In any case, the finding that endogenous NGB expression increases RGC resistance to glaucomatous damage may open new therapeutic perspectives for glaucoma and other neurodegenerative disorders.

The beneficial effects of NGB in neurodegeneration are mediated, at least in part, by the preservation of mitochondrial function and amelioration of oxidative stress. Ocular hypertension induced energy failure and oxidative stress in RGCs at day 7 after IOP elevation, as revealed by the fluorescence signal of DHE. When oxidized, DHE forms ethidium, which intercalates with DNAs. Superoxide production occurs primarily in the mitochondria, and ethidium generated from oxidized DHE binds mitochondrial DNAs and yields cytoplasmic fluorescent signal. When ethidium is produced exceeding the binding capacity of mitochondrial DNAs, it binds genomic DNAs and gives rise to bright nuclear fluorescence signal. ${ }^{51}$ In the present study, RGCs of ocular hypertensive mice revealed primarily cytoplasmic staining of DHE, suggesting that glaucoma or elevation of IOP evokes only moderate superoxide production.

Elevated IOP has been proposed to cause hypoxic insult, which promotes mitochondrial depolarization and therefore affects ATP production. ${ }^{52,53}$ Intracellular ATP concentrations reflect a combination of production and consumption of energy-dependent cell processes. In addition, mitochondrial dysfunction increases ROS production. ${ }^{54,55}$ When the cellular production of ROS overwhelms the intrinsic antioxidant capacity, it causes oxidative stress and results in damage to DNAs, proteins, and lipids. ${ }^{54,56,57}$ This process is thought to contribute to the pathogenesis of a number of neurodegenerative disorders. $^{58-60}$ Emerging evidence supports the involvement of mitochondrial dysfunction and oxidative stress triggered by IOP elevation as a final common pathway of glaucomatous neurodegeneration. ${ }^{46}$ Importantly, overexpression of NGB attenuated these responses and concomitantly resulted in augmented recovery of mitochondrial respiration and reduced ROS formation. Our data demonstrating a parallel maintenance of ATP level and enhanced cell viability after IOP elevation in Ngb-Tg mice are consistent with an antioxidant role for NGB. Thus, our data support the notion that NGB acts to maintain mitochondrial function under the stressed condition.

Using purified RGC cultures, we further demonstrated in vitro that NGB protects RGCs against glutamate-induced neurotoxicity in a purified cell population, supporting a role of NGB as an endogenous neuroprotective agent in retinal neurons. Glutamate is a neurotransmitter essential for normal function of the retina; however, in instances of retinal injury or disease, such as glaucoma, increased concentrations of extracellular glutamate trigger excessive and prolonged activation of $\mathrm{N}$-methyl-D- 
aspartate (NMDA)-type glutamate receptors. ${ }^{61}$ The pathological activation of NMDA receptors leads to massive $\mathrm{Ca}^{2+}$ entry into the neuron, where the $\mathrm{Ca}^{2+}$ is thought to accumulate in the mitochondria and result in elevated levels of superoxide and ROS. This oxidative stress model is paralleled by mitochondrial $\mathrm{Ca}^{2+}$ overload and ATP crisis, in which $\mathrm{Ca}^{2+}$ overload overwhelms the capacity of the mitochondria to generate ATP, leading to energy failure in RGCs and cell death. NGB may act on a common oxidative pathway to protect RGCs against both glutamate-induced neurocytotoxicity in vitro and elevated-IOP-induced neurodegeneration in vivo. However, evidence is also accumulating that these three pathways (glutamate toxicity, oxidative stress, and energy failure) can propagate through distinctive and mutually exclusive signal transduction pathways to contribute to cell loss after injury. Thus, we cannot rule out the possibility that the activities of NGB are not limited to antioxidation or transport of molecular $\mathrm{O}_{2}$, but extend to other potentially neuroprotective actions.

Although it would be most interesting to perform functional assessments in the mouse model of glaucoma, this is technically difficult to achieve. In an attempt to measure ocular function, we performed visual evoked potential studies to assess the integrity of the visual pathway, including RGCs. Significant differences of RGC survival could be detected between Ngb-Tg and wild-type mice after IOP elevation, although no significant difference in the visual evoked potential amplitudes was noted. The finding is in agreement with a recent report that the decrease of RGC number does not correlate with the decrease of visual evoked potential amplitudes in DBA/2J mice. ${ }^{62}$ These findings suggest that remaining RGCs can exhibit compensatory effect on their input into the brain either by altering the intensity of action potentials or by forming greater numbers of synapses.

Although we have now demonstrated the neuroprotective effects of NGB in an experimental model of glaucoma, future elucidation of the regulatory mechanisms and molecular interactions of NGB in modulating mitochondrial function, oxidative stress and/or other signaling events remains important. Putative mechanisms of glaucomatous RGC death include oxidative damage, mitochondrial dysfunction, glutamate excitotoxicity, defects in axonal transport, growth factor deficiency, and vascular factors. Given the multiple and parallel mechanisms involved in neurodegeneration, modulation of a single specific pathway will likely yield only a partial benefit, as we observed in Ngb-Tg mice in the present study. A combined strategy that targets multiple factors in a concerted way to prevent RGC loss and neurodegeneration may thus be more beneficial in glaucoma. Future investigation of a role of NGB in other retinal and central nervous system neurodegenerative disease models may illuminate the biochemical pathways underlying the neuroprotective functions of NGB and regulating its expression and function.

In summary, our data indicate that NGB can ameliorate the damaging effects of ocular hypertension to RGCs. Neuroprotection by NGB against elevated IOP or glutamate might occur through decreasing oxidative stress and preserving the function of mitochondria. In addition to its use in dissecting pathophysiology, our newly generated Ngb-Tg mouse may also serve as a useful tool for elucidating the functional roles and mechanisms of NGB under physiological conditions. By exploring the potential mechanisms regulating RGC survival under stressed conditions, the results of this research may offer novel protective strategies to treat or prevent the degeneration of neurons and optic nerves in glaucoma patients.

\section{Acknowledgments}

We thank Marie Ortega for animal care and Don Pottle for help with confocal microscopy.

\section{References}

1. Burmester $T$, Weich $B$, Reinhardt $S$, Hankeln $T$ : A vertebrate globin expressed in the brain. Nature 2000, 407:520-523

2. Zhang CG, Li L, Deng MY, Xie F, Wang CL, Zhou WQ, Wang HY, He FC: [Coding region cDNA sequence cloning of rat neuroglobin gene, its polymorphism feature and tissue expression profile analysis]. Chinese. Yi Chuan Xue Bao 2001, 28:997-1001

3. Pesce A, Bolognesi M, Bocedi A, Ascenzi P, Dewilde S, Moens L, Hankeln T, Burmester T: Neuroglobin and cytoglobin. Fresh blood for the vertebrate globin family. EMBO Rep 2002, 3:1146-1151

4. Brunori M, Vallone B: A globin for the brain. FASEB J 2006, 20:21922197

5. Wystub S, Laufs T, Schmidt M, Burmester T, Maas U, Saaler-Reinhardt S, Hankeln T, Reuss S: Localization of neuroglobin protein in the mouse brain. Neurosci Lett 2003, 346:114-116

6. Fordel E, Geuens E, Dewilde S, De Coen W, Moens L: Hypoxia/ ischemia and the regulation of neuroglobin and cytoglobin expression. IUBMB Life 2004, 56:681-687

7. Reuss S, Saaler-Reinhardt S, Weich B, Wystub S, Reuss MH, Burmester T, Hankeln T: Expression analysis of neuroglobin mRNA in rodent tissues. Neuroscience 2002, 115:645-656

8. Schmidt M, Giessl A, Laufs T, Hankeln T, Wolfrum U, Burmester T: How does the eye breathe? Evidence for neuroglobin-mediated oxygen supply in the mammalian retina. J Biol Chem 2003, 278:19321935

9. Rajendram R, Rao NA: Neuroglobin in normal retina and retina from eyes with advanced glaucoma. Br J Ophthalmol 2007, 91:663-666

10. Garry DJ, Mammen PP: Neuroprotection and the role of neuroglobin. Lancet 2003, 362:342-343

11. Greenberg DA, Jin K, Khan AA: Neuroglobin: an endogenous neuroprotectant. Curr Opin Pharmacol 2008, 8:20-24

12. Yu Z, Fan $X$, Lo EH, Wang $X$ : Neuroprotective roles and mechanisms of neuroglobin. Neurol Res 2009, 31:122-127

13. Ostojić J, Sakaguchi DS, de Lathouder Y, Hargrove MS, Trent JT 3rd, Kwon YH, Kardon RH, Kuehn MH, Betts DM, Grozdanić S: Neuroglobin and cytoglobin: oxygen-binding proteins in retinal neurons. Invest Ophthalmol Vis Sci 2006, 47:1016-1023

14. Ostojić J, Grozdanić S, Syed NA, Hargrove MS, Trent JT 3rd, Kuehn $\mathrm{MH}$, Kardon $\mathrm{RH}$, Kwon YH, Sakaguchi DS: Neuroglobin and cytoglobin distribution in the anterior eye segment: a comparative immunohistochemical study. J Histochem Cytochem 2008, 56:863-872

15. Ostojić J, Grozdanić S, Syed NA, Hargrove MS, Trent JT 3rd, Kuehn $\mathrm{MH}$, Kwon YH, Kardon RH, Sakaguchi DS: Patterns of distribution of oxygen-binding globins, neuroglobin and cytoglobin in human retina. Arch Ophthalmol 2008, 126:1530-1536

16. Nienhaus K, Nienhaus GU: Searching for neuroglobin's role in the brain. IUBMB Life 2007, 59:490-497

17. Burmester T, Hankeln T: What is the function of neuroglobin? J Exp Biol 2009, 212:1423-1428

18. Herold S, Fago A, Weber RE, Dewilde S, Moens L: Reactivity studies of the $\mathrm{Fe}(\mathrm{III})$ and $\mathrm{Fe}(\mathrm{II}) \mathrm{NO}$ forms of human neuroglobin reveal a potential role against oxidative stress. J Biol Chem 2004, 279:2284122847 
19. Rayner BS, Duong TT, Myers SJ, Witting PK: Protective effect of a synthetic anti-oxidant on neuronal cell apoptosis resulting from experimental hypoxia re-oxygenation injury. J Neurochem 2006, 97 211-221

20. Fordel E, Thijs L, Martinet W, Schrijvers D, Moens L, Dewilde S: Anoxia or oxygen and glucose deprivation in SH-SY5Y cells: a step closer to the unraveling of neuroglobin and cytoglobin functions. Gene 2007, 398:114-122

21. Brunori M, Giuffrè A, Nienhaus K, Nienhaus GU, Scandurra FM, Vallone B: Neuroglobin, nitric oxide, and oxygen: functional pathways and conformational changes. Proc Natl Acad Sci USA 2005, 102 8483-8488

22. Wakasugi K, Nakano T, Morishima I: Oxidized human neuroglobin acts as a heterotrimeric Galpha protein guanine nucleotide dissociation inhibitor. J Biol Chem 2003, 278:36505-36512

23. Wakasugi K, Morishima I: Preparation and characterization of a chimeric zebrafish-human neuroglobin engineered by module substitution. Biochem Biophys Res Commun 2005, 330:591-597

24. Sun Y, Jin K, Mao XO, Zhu Y, Greenberg DA: Neuroglobin is upregulated by and protects neurons from hypoxic-ischemic injury. Proc Natl Acad Sci USA 2001, 98:15306-15311

25. Sun Y, Jin K, Peel A, Mao XO, Xie L, Greenberg DA: Neuroglobin protects the brain from experimental stroke in vivo. Proc Natl Acad Sci USA 2003, 100:3497-3500

26. Peroni D, Negro A, Bähr M, Dietz GP: Intracellular delivery of neuroglobin using HIV-1 TAT protein transduction domain fails to protect against oxygen and glucose deprivation. Neurosci Lett 2007, 421: $110-114$

27. Hundahl C, Kelsen J, Kjaer K, Rønn LC, Weber RE, Geuens E, Hay-Schmidt A, Nyengaard JR: Does neuroglobin protect neurons from ischemic insult? A quantitative investigation of neuroglobin expression following transient MCAo in spontaneously hypertensive rats. Brain Res 2006, 1085:19-27

28. Mackenzie P, Cioffi G: How does lowering of intraocular pressure protect the optic nerve? Surv Ophthalmol 2008, 53 Suppl 1:S39-S43

29. Mozaffarieh M, Flammer J: Is there more to glaucoma treatment than lowering IOP? Surv Ophthalmol 2007, 52 Suppl 2:S174-S179

30. Mozaffarieh M, Flammer J: A novel perspective on natural therapeutic approaches in glaucoma therapy. Expert Opin Emerg Drugs 2007 12:195-198

31. Liu Q, Ju WK, Crowston JG, Xie F, Perry G, Smith MA, Lindsey JD, Weinreb RN: Oxidative stress is an early event in hydrostatic pressure induced retinal ganglion cell damage. Invest Ophthalmol Vis Sci 2007, 48:4580-4589

32. Ju WK, Kim KY, Angert M, Duong-Polk KX, Lindsey JD, Ellisman MH, Weinreb RN: Memantine blocks mitochondrial OPA1 and cytochrome $\mathrm{c}$ release and subsequent apoptotic cell death in glaucomatous retina. Invest Ophthalmol Vis Sci 2009, 50:707-716

33. Nucci C, Tartaglione R, Rombolà L, Morrone LA, Fazzi E, Bagetta G: Neurochemical evidence to implicate elevated glutamate in the mechanisms of high intraocular pressure (IOP)-induced retinal ganglion cell death in rat. Neurotoxicology 2005, 26:935-941

34. Russo R, Rotiroti D, Tassorelli C, Nucci C, Bagetta G, Bucci MG, Corasaniti MT, Morrone LA: Identification of novel pharmacological targets to minimize excitotoxic retinal damage. Int Rev Neurobiol 2009, 85:407-423

35. Saccà SC, Izzotti A, Rossi P, Traverso C: Glaucomatous outflow pathway and oxidative stress. Exp Eye Res 2007, 84:389-399

36. Tezel G: Oxidative stress in glaucomatous neurodegeneration: mechanisms and consequences. Prog Retin Eye Res 2006, 25:490-513

37. Sappington RM, Carlson BJ, Crish SD, Calkins DJ: The microbead occlusion model: a paradigm for induced ocular hypertension in rats and mice. Invest Ophthalmol Vis Sci 2010, 51:207-216

38. Chen H, Wei X, Cho KS, Chen G, Sappington R, Calkins DJ, Chen DF: Optic neuropathy due to microbead-induced elevated intraocular pressure in the mouse. Invest Ophthalmol Vis Sci 2011, 52:36-44

39. Wang X, Liu J, Zhu H, Tejima E, Tsuji K, Murata Y, Atochin DN, Huang PL, Zhang C, Lo EH: Effects of neuroglobin overexpression on acute brain injury and long-term outcomes after focal cerebral ischemia. Stroke 2008, 39:1869-1874
40. Cho KS, Yang L, Lu B, Feng Ma H, Huang X, Pekny M, Chen DF: Re-establishing the regenerative potential of central nervous system axons in postnatal mice. J Cell Sci 2005, 118:863-872

41. Huang X, Wu DY, Chen G, Manji H, Chen DF: Support of retina ganglion cell survival and axon regeneration by lithium through a Bcl-2-dependent mechanism. Invest Ophthalmol Vis Sci 2003, 44: 347-354

42. Jiao J, Huang X, Feit-Leithman RA, Neve RL, Snider W, Dartt DA, Chen DF: Bcl-2 enhances $\mathrm{Ca}(2+)$ signaling to support the intrinsic regenerative capacity of CNS axons. EMBO J 2005, 24:1068-1078

43. Koprivica V, Cho KS, Park JB, Yiu G, Atwal J, Gore B, Kim JA, Lin E, Tessier-Lavigne M, Chen DF, He Z: EGFR activation mediates inhibition of axon regeneration by myelin and chondroitin sulfate proteoglycans. Science 2005, 310:106-110

44. Levkovitch-Verbin H, Quigley HA, Martin KR, Valenta D, Baumrind LA Pease ME: Translimbal laser photocoagulation to the trabecular meshwork as a model of glaucoma in rats. Invest Ophthalmol Vis Sci 2002, 43:402-410

45. Seki M, Lipton SA: Targeting excitotoxic/free radical signaling pathways for therapeutic intervention in glaucoma. Prog Brain Res 2008 173:495-510

46. Kong GY, Van Bergen NJ, Trounce IA, Crowston JG: Mitochondrial dysfunction and glaucoma. J Glaucoma 2009, 18:93-100

47. Del Rio D, Stewart AJ, Pellegrini N: A review of recent studies on malondialdehyde as toxic molecule and biological marker of oxidative stress. Nutr Metab Cardiovasc Dis 2005, 15:316-328

48. Fan B, Li GY, Li YP, Cui JZ: [Neuroprotective effect of epigallocatechin gallate on oxidative-stress-injured retinal cells]. Chinese. Zhonghua Yi Xue Za Zhi 2008, 88:1711-1714

49. Liu J, Yu Z, Guo S, Lee SR, Xing C, Zhang C, Gao Y, Nicholls DG, Lo $\mathrm{EH}$, Wang $X$ : Effects of neuroglobin overexpression on mitochondrial function and oxidative stress following hypoxia/reoxygenation in cultured neurons. J Neurosci Res 2009, 87:164-170

50. Schmidt M, Laufs T, Reuss S, Hankeln T, Burmester T: Divergent distribution of cytoglobin and neuroglobin in the murine eye. Neurosc Lett 2005, 374:207-211

51. Budd SL, Castilho RF, Nicholls DG: Mitochondrial membrane potential and hydroethidine-monitored superoxide generation in cultured cerebellar granule cells. FEBS Lett 1997, 415:21-24

52. Anderson MF, Sims NR: Mitochondrial respiratory function and cell death in focal cerebral ischemia. J Neurochem 1999, 73:1189-1199

53. Chen K, Zhang Q, Wang J, Liu F, Mi M, Xu H, Chen F, Zeng K: Taurine protects transformed rat retinal ganglion cells from hypoxia-induced apoptosis by preventing mitochondrial dysfunction. Brain Res 2009, 1279:131-138

54. Howell N: LHON and other optic nerve atrophies: the mitochondrial connection. Dev Ophthalmol 2003, 37:94-108

55. Takahashi T, Kitaoka K, Ogawa Y, Kobayashi T, Seguchi H, Tani T, Yoshida S: Lysosomal dysfunction on hydrogen peroxide-induced apoptosis of osteoarthritic chondrocytes. Int J Mol Med 2004 14:197-200

56. Melov S: Spotlight on nematode mitochondria in RNAi mega-screen Sci Aging Knowledge Environ 2002, 2002(47):pe18

57. Negi G, Kumar A, Kaundal RK, Gulati A, Sharma SS: Functional and biochemical evidence indicating beneficial effect of melatonin and nicotinamide alone and in combination in experimental diabetic neuropathy. Neuropharmacology 2010, 58:585-592

58. Perez-Gracia E, Blanco R, Carmona M, Carro E, Ferrer I: Oxidative stress damage and oxidative stress responses in the choroid plexus in Alzheimer's disease. Acta Neuropathol 2009, 118:497-504

59. Gallagher DA, Schapira AH: Etiopathogenesis and treatment of Parkinson's disease. Curr Top Med Chem 2009, 9:860-868

60. Roberts RA, Laskin DL, Smith CV, Robertson FM, Allen EM, Doorn JA, Slikker W: Nitrative and oxidative stress in toxicology and disease. Toxicol Sci 2009, 112:4-16

61. Lipton SA: Pathologically-activated therapeutics for neuroprotection: mechanism of NMDA receptor block by memantine and S-nitrosylation. Curr Drug Targets 2007, 8:621-632

62. Heiduschka P, Julien S, Schuettauf F, Schnichels: Loss of retinal function in aged DBA/2J mice - new insights into retinal neurodegeneration. Exp Eye Res 2010, 91:779-783 